\title{
Blinded predictions of standard binding free energies: lessons learned from the SAMPL6 challenge
}

\author{
Michail Papadourakis $^{1} \cdot$ Stefano Bosisio $^{1} \cdot$ Julien Michel $^{1}$ iD
}

Received: 2 June 2018 / Accepted: 24 August 2018 / Published online: 29 August 2018

(c) The Author(s) 2018

\begin{abstract}
In the context of the SAMPL6 challenges, series of blinded predictions of standard binding free energies were made with the SOMD software for a dataset of 27 host-guest systems featuring two octa-acids hosts (OA and TEMOA) and a cucurbituril ring ( $C B 8$ ) host. Three different models were used, ModelA computes the free energy of binding based on a double annihilation technique; ModelB additionally takes into account long-range dispersion and standard state corrections; ModelC additionally introduces an empirical correction term derived from a regression analysis of SAMPL5 predictions previously made with SOMD. The performance of each model was evaluated with two different setups; buffer explicitly matches the ionic strength from the binding assays, whereas no-buffer merely neutralizes the host-guest net charge with counter-ions. ModelC/no-buffer shows the lowest mean-unsigned error for the overall dataset (MUE $1.29<1.39<1.50 \mathrm{kcal} \mathrm{mol}^{-1}$, 95\% $\mathrm{CI}$ ), while explicit modelling of the buffer improves significantly results for the CB8 host only. Correlation with experimental data ranges from excellent for the host TEMOA $\left(\mathrm{R}^{2} 0.91<0.94<0.96\right)$, to poor for $C B 8\left(\mathrm{R}^{2} 0.04<0.12<0.23\right)$. Further investigations indicate a pronounced dependence of the binding free energies on the modelled ionic strength, and variable reproducibility of the binding free energies between different simulation packages.
\end{abstract}

Keywords SAMPL6 $\cdot$ SAMPLing $\cdot$ Binding free energy $\cdot$ Alchemical free energy

\section{Introduction}

Computer-aided drug design (CADD) is a powerful methodology for early stage drug-discovery [1]. In particular there is much interest in the use of molecular simulations methods to support drug-discovery efforts [2], via for instance investigation of protein folding mechanisms [3, 4], or ligand modulation of millisecond time-scale conformational changes in proteins [5]. Another application of molecular simulations in CADD is potency predictions to decrease time and costs of hit-to-lead and lead optimization stages needed before molecules may be progressed towards clinical studies [6]. This requires accurate description of ligand-protein energetics,

Electronic supplementary material The online version of this article (https://doi.org/10.1007/s10822-018-0154-6) contains supplementary material, which is available to authorized users.

Julien Michel

mail@julienmichel.net

1 EaStCHEM School of Chemistry, Joseph Black Building, King's Buildings, Edinburgh EH9 3FJ, UK which is nowadays increasingly sought via use of free energy calculations methods.

Among various existing free energy calculation methodologies, alchemical free energy calculations (AFE) have attracted much interest in recent years [7-9], due to their robust grounding in statistical physics. AFE calculations capture non-additivity of structure-activity relationship in congeneric series that are overlooked by empirical scoring methods [10], and have given useful potency estimates for a range of protein-ligand systems [11-13]. AFE methods may also be used to predict physical properties, such as lipophilicity coefficients [14-16]. In spite of encouraging successes, there are still important technical hurdles to overcome. Usual concerns involve finite-sampling effects that introduce statistical errors [17-20], whereas inaccuracies in potential energy functions contribute to systematic errors [21]. Additionally, algorithmic decisions for the handling of long range electrostatic interactions and finite-size artefacts affect simulation results in ways that are still poorly understood, with effects particularly apparent in the modelling of charged species [22-24]. Thus, it is important to improve the 


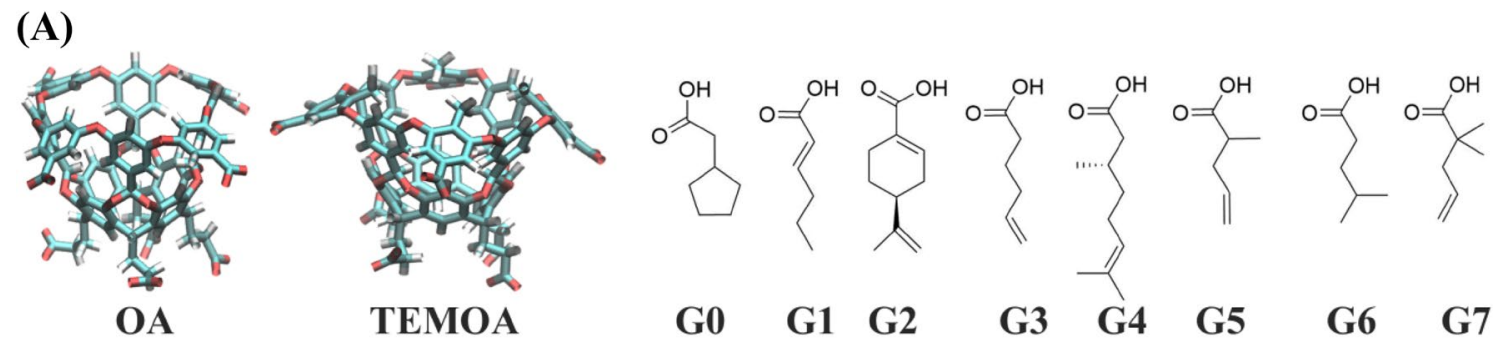

(B)

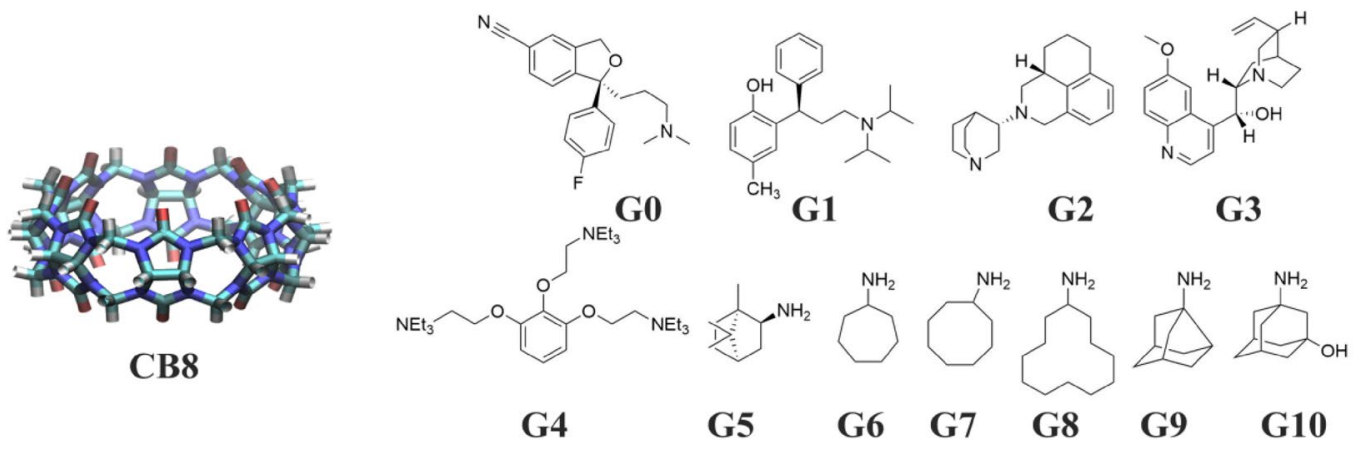

Fig. 1 Depiction of the SAMPL6 host-guest dataset. a OA and TEMOA host-guest systems. b CB8 host-guest systems

robustness of AFE protocols to enable their reliable application to structure-based drug design problems.

Blinded prediction competitions offer a valuable resource to reduce bias in validation studies and to test practical utility of a methodology in a setting that more closely resembles CADD in practice [25]. The D3R Grand Challenges have proven a popular blinded competition, with a focus on validating computational methods for modelling of protein-ligand interactions [25, 26]. The Statistical Assessment of Modelling of Proteins and Ligands (SAMPL) is also a well-established blinded competition for free energy science in drug discovery [27]. The SAMPL challenge was founded in 2007 and usually requests participants to predict physical chemical properties, such as binding affinities for host-guest systems, or hydration free energies of small drug-like molecules [28, 29]. Host-guest systems are attractive since they provide more tractable milestones towards validation of protocols for modelling protein-ligand binding energetics [30].

The 6th SAMPL (SAMPL6) competition was launched in September 2017. Our group focused on the host-guest leg of this contest, which requested predictions of standard free energies of binding for 27 guests across three different hosts [31]. The host molecules consisted in two octa-acids, $O A$ and TEMOA molecules [32-35], and a cucurbituril ring clip $C B 8$ [36-39], as shown in Fig. 1. The octa-acid systems (Fig. 1a) are basket shaped; $O A$ contains four flexible propionate side chains bearing two rotatable single bonds each, while TEMOA contains four methyl groups, which alter the shape of the hydrophobic cavity. $C B 8$ (Fig. 1b) is a heteroaromatic multicyclic molecule, chemically related to the cucurbiturils, made of methylene bridges containing eight glycoluril units. $C B 8$ is considered a more flexible host than $O A$ and TEMOA, though the latter two also contain flexible groups at the top and bottom of their cavities [38, 39]. Additionally, SAMPL6 introduced a SAMPLing challenge focused on evaluating convergence and reproducibility (across codes) of free energy predictions. To this end, input files for parameterized host-guests $O A-G 3, O A-G 6$ and $C B 8-G 3$ were provided and participants requested to evaluate convergence of their binding free energy estimates.

This report summarizes the performance of our free energy code Sire/OpenMM Molecular Dynamics (SOMD) against the SAMPL6 host-guest dataset, as well as the lessons learned for continuing efforts to improve the robustness of AFE methods in CADD.

\section{Theory and methods}

\section{Definition of binding affinity}

The reversible binding of a ligand $L$ to a receptor $P$ can be written as:

$P+L \stackrel{\Delta G_{\text {bind }}^{\circ}}{\rightleftharpoons} P L$

where $\Delta G^{\circ}{ }_{b i n d}$ is the standard free energy of binding of ligand $L$ to receptor $P$. A statistical thermodynamics treatment leads to Eq. 2 [40]: 
Fig. 2 Thermodynamic cycle for standard binding free energy calculations. Firstly, the fully interacting guest is simulated in a free phase (top left) and a bound phase (top right), then the charges and the van der Waals terms are switched off, resulting in a non-interacting guest in water (bottom left), and bound to the host (bottom right)

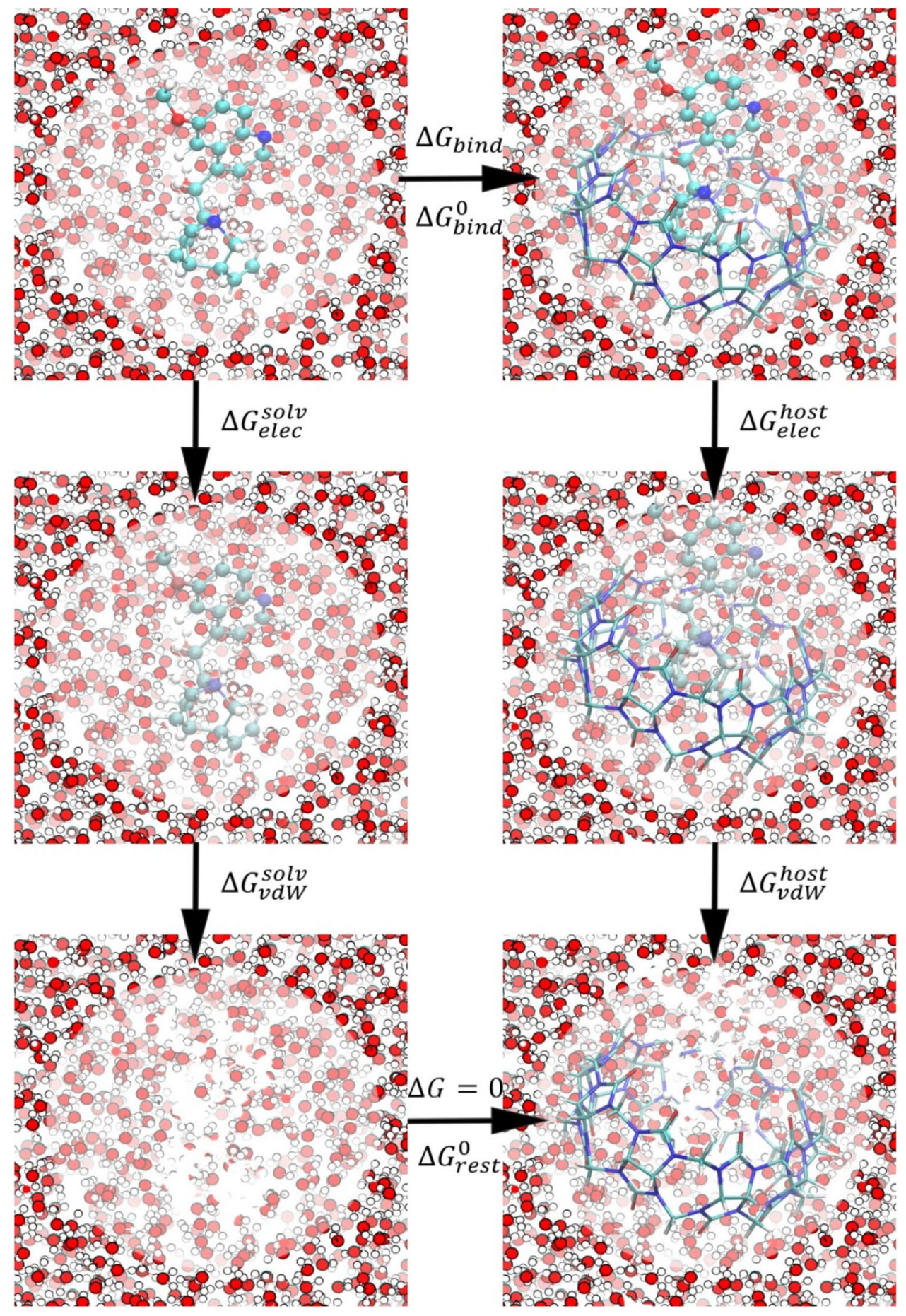

$\Delta G_{\text {bind }}^{o}=-k_{B} T \ln \frac{Z_{P L, \text { solv }} Z_{\text {solv }} V}{Z_{L, \text { solv }} Z_{P, \text { solv }} V_{o}}$

where $Z_{P L \text {,solv }}, Z_{\text {solv }}, Z_{L \text {,solv }}$ and $Z_{P \text {,solv }}$ are the configuration integrals for complex system, the solvent molecules, the ligand and the protein system respectively, $V$ is the volume of binding, namely the volume available to the ligand to bind the protein, and $V_{o}$ the standard state volume, which is usually equal to $1661 \AA^{3} /$ molecule.

\section{Computing free energies of binding through models A, B, C}

Equation 2 can be applied to estimate the binding free energy for host-guest systems. Computationally, the free 
energy is evaluated by using molecular dynamics simulations (MD) by means of a double annihilation technique [13, 41, 42]. Figure 2 shows how this approach is used to evaluate $\Delta G_{\text {bind }}^{\circ}$ by means of a thermodynamic cycle. In the first step (discharging step) the charges of the guest's atoms are turned off both in the solvated phase and in the bound phase, providing the discharging free energy changes $\Delta G_{\text {elec }}^{\text {solv }}$ and $\Delta G_{\text {elec }}^{\text {host }}$ respectively. In the second step (vanishing step) a "non-interacting" guest is obtained by now switching off the van der Waals parameters of the discharged guest both in solvent and complex phase, giving the vanishing free energy changes, $\Delta G_{v d W}^{s o l v}$ and $\Delta G_{v d W}^{\text {host }}$, respectively. To prevent the ligand from drifting away from the host cavity a series of a flat-bottom distance restraints are defined between one guest atom $j$ closest to the center of mass of the guest and four host atoms $i$. The restraint potential is given by Eq. 3 [13]:

$U_{\left(d_{j 1}, \ldots, d_{\left.j N_{\text {host }}\right)}^{\text {restr }}\right)}^{N_{\text {host }}} \begin{cases}0 & \text { if }\left|d_{j i}-R_{j i}\right| \leq D_{j i} \\ \kappa_{i j}\left(\left|d_{j i}-R_{j i}\right|-D_{j i}\right)^{2} & \text { if }\left|d_{j i}-R_{j i}\right|>D_{j i}\end{cases}$

where $U_{\left(d_{j 1}, \ldots, d_{j N_{h o s}}\right)}^{\text {restr }}$ is the potential energy of the restraint as a function between a guest atom $j$ and a set of host atoms $i$, lol denotes the absolute value, $D_{j i}$ is the restraint deviation tolerance, $R_{j i}$ is the reference distance between host and guest atom, $\kappa_{j i}$ is the restraint force constant and $N_{\text {host }}$ is the number of host atoms that contribute to the restraint.

From the closure of the thermodynamic cycle (Fig. 2) the binding free energy $\Delta G_{\text {bind }}$ is given by Eq. 4 :

$\Delta G_{\text {bind }}^{\text {ModelA }}=\left(\Delta G_{\text {elec }}^{\text {solv }}+\Delta G_{v d W}^{\text {solv }}\right)-\left(\Delta G_{\text {elec }}^{\text {host }}+\Delta G_{v d W}^{\text {host }}\right)$

Free energies of binding computed with Eq. 4 will be referred to as ModelA binding energies.

ModelA does not take into account the contribution of long range dispersions interactions due to the use of nonbonded cutoffs. Thus, to improve over ModelA, a long-range dispersion correction term is added to the free energy of binding by post-processing of the end states trajectories [43]. Additionally, a free energy correction term is introduced to relate the volume available to the restrained but non-interacting ligand to standard state conditions. This leads to Eq. 5 for predictions of binding free energies via ModelB.

$\Delta G_{\text {bind }}^{0, \text { ModelB }}=\Delta G_{\text {bind }}^{\text {ModelA }}+\left(\Delta G_{L J L R C}^{\text {host }}-\Delta G_{\text {LJLRC }}^{\text {solv }}\right)+\Delta G_{\text {restr }}^{0}$

$\Delta G_{L J L R C}^{\text {host }}$ is the long range correction term for the bound phase, and $\Delta G_{L J L R C}^{\text {solv }}$ is the LRC term for the solvated phase. Details for the evaluation of these terms have been provided elsewhere [13]. $\Delta G_{\text {restr }}^{0}$ is the free energy cost for imposing the host-guest restraint which is given by Eq. 6 :
$\Delta G_{\text {restr }}^{0}=-k_{B} T \ln \left(\frac{Z_{H \cdot G_{\text {ideal }}}}{Z_{H, \text { solv }} Z_{G, \text { gas }}}\right)$

where $Z_{H \cdot \cdot G_{\text {ideal }}}$ is the configuration integral for the restrained decoupled guest bound to the host, $Z_{H, \text { solv }}$ is the configuration integral for the solvated host and $Z_{G \text {,gas }}$ is the configuration integral for the guest in an ideal thermodynamic state. Equation 6 is evaluated by numerical integration as described elsewhere [13].

Finally, ModelC was constructed by devising an empirical correction term to account for systematic errors due to finite size artefacts and inaccuracies in potential energy functions. Linear regression models were obtained by correlating past SAMPL5 binding free energies computed with SOMD to experimental data, leading to Eq. 7 to compute ModelC binding free energies:

$\Delta G_{\text {bind }}^{0, \text { ModelC }}=\frac{\Delta G_{\text {bind }}^{0, \text { ModelB }}-\beta}{\alpha}$

where $\alpha$ and $\beta$ are the slope and intercept of the linear regression model. SAMPL5 featured the same hosts OA and TEMOA but a different host CB7. Thus, separate regression models were determined for use with OA, TEMOA or CB8 hosts, the parameters are given in Table S1.

\section{Preparation of host-guest input files for free energy calculations}

The SAMPL6 organizers provided mol2 files for hosts, $O A$, TEMOA and $C B 8$, and ligands, depicted in Fig. 1. Each file had the same Cartesian frame of reference and docking was performed with OpenEye toolkit [44-46] to predict the most likely binding mode. Experimental measurements were done at a $\mathrm{pH} 11.7 \pm 0.1$ at $298 \mathrm{~K}$ in presence of a buffer of $10 \mathrm{mM} \mathrm{Na}_{3} \mathrm{PO}_{4}$ for $O A$ and TEMOA. $C B 8$ was measured at $\mathrm{pH} 7.4 \pm 0.1$ at $298 \mathrm{~K}$ with $25 \mathrm{mM} \mathrm{Na}_{3} \mathrm{PO}_{4}$ buffer. To understand the influence of the buffer on binding free energy predictions, two different sets of input files were prepared, leading to no-buffer and buffer setups.

\section{Input files for the no-buffer setup}

In the no-buffer simulations, the presence of the additional $\mathrm{Na}_{3} \mathrm{PO}_{4}$ buffer was neglected. OA, TEMOA and $C B 8$ host-guest systems were parametrized starting from the mol2 host and guest's files. The force field parameters for $O A$ and TEMOA hosts were taken from a preceding study of host-guest binding energies carried out for the SAMPL5 contest [13]. To create the host-guest complex input files, the utilities parmed and tleap were used [47, 48]. The combined host-guest complex mol2 file was loaded in tleap 
along with host force field parameters and GAFF1.8 and AM1/BCC parameters for the ligand as generated by antechamber from the AMBER16 release [49, 50]. The system was solvated in a cubic box with TIP3P water molecules [51], with a minimum distance between the solute and the box of $12 \AA$. Counter ions were added to neutralize the total net charge. The same approach was followed for parameterizing the ligand in a solvated phase.

Next an equilibration protocol was applied to relax the box size. Initially, energy minimization of the entire system was performed with 100 steps of steepest descent gradients, using sander. Then, solute molecules were position restrained with a force constant of $10 \mathrm{kcal} \mathrm{mol}^{-1} \AA^{-2}$ while water molecules were allowed to equilibrate in an NVT ensemble, 200 ps at $298 \mathrm{~K}$, followed by a NPT equilibration for further $200 \mathrm{ps}$ at $1 \mathrm{~atm}$ pressure. Finally, a 2 ns NPT MD simulation was run with the $S O M D$ software (revision 2017.1.0) to reach a final density of about $1 \mathrm{~g} \mathrm{~cm}^{-3}$ [52, 53]. The final coordinate files were retrieved with cpptraj. The edge length of the host-guest boxes was about $50 \AA$, whereas the solvated guest phase had an edge length of about $35 \AA$.

\section{Input files for the buffer setup}

For the second set of simulations, additional counter ions were added to mimic the presence of a buffer in the experiments. However, $\mathrm{Na}_{3} \mathrm{PO}_{4}$ was modelled by $\mathrm{NaCl}$ as force field parameters for multivalent ions were not readily available. Thus, for $O A$ and TEMOA systems, the $10 \mathrm{mM}$ sodium phosphate buffer was modelled with $60 \mathrm{mM}$ of $\mathrm{NaCl}$ to match the ionic strength of the solution used for the experiments. Starting from the complex phase files, created as described previously, 4 additional $\mathrm{Na}^{+}$and $4 \mathrm{Cl}^{-}$ions were added to each system, using tleap. The equilibration protocol described previously was reapplied to adjust the placement of the counter ions. For the preparation of the solvated phase, the host molecule was extracted from an equilibrated host-guest box and the host's heavy atoms were replaced with water molecules. After equilibration the final solvated phase system had the same amount of $\mathrm{Na}^{+}$and $\mathrm{Cl}^{-}$ions as in the host-guest complex system, and a similar box size dimension. The same procedure was followed for $C B 8$. In this case, $25 \mathrm{mM} \mathrm{Na}_{3} \mathrm{PO}_{4}$ were matched with $150 \mathrm{mM}$ $\mathrm{NaCl}$, thus $8 \mathrm{Na}^{+}$and $8 \mathrm{Cl}^{-}$ions were added to each $C B 8$ host-guest system.

\section{SAMPL6 simulation protocols}

For the octa-acid hosts, both complex and solvated phase discharging step were run with nine equidistant $\lambda$ windows. Twelve $\lambda$ windows $(0.0,0.1,0.2,0.3,0.4,0.5,0.6,0.7,0.8$, $0.9,0.95,1.0)$ were employed for the vanishing step, both in bound and solvated phase. For the $C B 8$ host the bound and solvated phase discharging steps have been run with nine equidistant $\lambda$ windows. The solvated vanishing step was carried out with the same windows setup as for the octa-acid guests. The bound vanishing step was carried out with 16 $\lambda$ windows $(\lambda 0.00,0.05,0.10,0.15,0.20,0.25,0.30,0.35$, $0.40,0.45,0.50,0.55,0.60,0.70,0.85,1.00)$ as preliminary runs indicated a need for greater number of windows to obtain reliable free energy changes.

All the simulations were run for duration of $8 \mathrm{~ns}$ with SOMD in an NPT ensemble. Temperature control was achieved with an Andersen Thermostat with a coupling constant of $10 \mathrm{ps}^{-1}$ [54]. Pressure control was maintained by a Monte Carlo barostat that attempted isotropic box edge scaling every 100 fs. A $12 \AA$ atom-based cutoff distance for the non-bonded interactions was used, using a Barker Watts reaction field with dielectric constant of 78.3 [55]. In the bound phase the restraints parameters of Eq. 3 were: $R_{j i}=5 \AA, D_{j i}=2 \AA$ and $\kappa_{i j}=10 \mathrm{kcal} \mathrm{mol}^{-1} \AA^{-2}$ for all the octa-acid systems, while $R_{j i}=7 \AA, D_{j i}=2 \AA$ and $\kappa_{j i}=10 \mathrm{kcal}$ $\mathrm{mol}^{-1} \AA^{-2}$ were chosen for the $C B 8$ simulations The guest atom $j$ was taken as the atom closest to the center of mass of the guest. The atom names in the input files were for $\mathrm{OA}$ : $\mathrm{G} 0=\mathrm{C} 6 ; \mathrm{G} 1=\mathrm{C} 2 ; \mathrm{G} 2=\mathrm{C} 9 ; \mathrm{G} 3=\mathrm{C} 6 ; \mathrm{G} 4=\mathrm{C} 1 ; \mathrm{G} 5=\mathrm{C} 5$; $\mathrm{G} 6=\mathrm{C} 6 ; \mathrm{G} 7=\mathrm{C} 6$. For TEMOA: $\mathrm{G} 1=\mathrm{C} 5 ; \mathrm{G} 2=\mathrm{C} 9$; $\mathrm{G} 3=\mathrm{C} 6 ; \mathrm{G} 4=\mathrm{C} 7 ; \mathrm{G} 5=\mathrm{C} 5 ; \mathrm{G} 6=\mathrm{C} 6 ; \mathrm{G} 7=\mathrm{C} 6$. The four host atom names $i$ in OA and TEMOA were C45, C51, C57, C63. The selection of $R_{\mathrm{ij}}$ and $D_{\mathrm{ij}}$ parameters were based on an average distance of $4.9 \AA$ measured between these four host atoms and the guest atom C6 in G0 in the input files provided by the organizers. For CB8 the guest and host atom names were: $\mathrm{G} 0=(\mathrm{C} 11, \mathrm{C} 3, \mathrm{C} 10, \mathrm{C} 18, \mathrm{C} 26), \mathrm{G} 1=(\mathrm{C} 20$, $\mathrm{C} 4, \mathrm{C} 12, \mathrm{C} 22, \mathrm{C} 31), \mathrm{G} 2=(\mathrm{C} 13, \mathrm{C} 8, \mathrm{C} 18, \mathrm{C} 26, \mathrm{C} 32)$, $\mathrm{G} 3=(\mathrm{C} 18, \mathrm{C} 2, \mathrm{C} 10, \mathrm{C} 16, \mathrm{C} 24), \mathrm{G} 4=(\mathrm{C} 5, \mathrm{C} 4, \mathrm{C} 10, \mathrm{C} 16$, $\mathrm{C} 24), \mathrm{G} 5=(\mathrm{C} 7, \mathrm{C} 6, \mathrm{C} 14, \mathrm{C} 22, \mathrm{C} 28), \mathrm{G} 6=(\mathrm{C} 5, \mathrm{C} 6, \mathrm{C} 14$, $\mathrm{C} 22, \mathrm{C} 32), \mathrm{G} 7=(\mathrm{C} 6, \mathrm{C} 4, \mathrm{C} 10, \mathrm{C} 16, \mathrm{C} 24), \mathrm{G} 8=(\mathrm{C} 10, \mathrm{C} 4$, $\mathrm{C} 14, \mathrm{C} 20, \mathrm{C} 26), \mathrm{G} 9=(\mathrm{C} 6, \mathrm{C} 4, \mathrm{C} 12, \mathrm{C} 20, \mathrm{C} 28), \mathrm{G} 10=(\mathrm{C} 7$, $\mathrm{C} 2, \mathrm{C} 10, \mathrm{C} 16, \mathrm{C} 24)$. The selection of $R_{\mathrm{ij}}$ and $D_{\mathrm{ij}}$ parameters were based on an average distance of $6.6 \AA$ measured between the four host atoms and the guest atom in G0 in the input files provided by the organizers.

\section{SAMPLing simulation protocols}

For the SAMPLing leg of the challenge topologies and coordinate file for five replicates of $O A-G 3, \mathrm{OA}-\mathrm{G} 6$ and $C B 8-G 3$ were provided by the organizers for both the complex phase and the solvated phase simulations. All simulations were run for duration of $20 \mathrm{~ns}$ per window with SOMD with other simulation parameters identical to those used for SAMPL6 unless otherwise mentioned. 


\section{Estimation of free energy of binding and evaluation of dataset metrics}

Free energy changes were computed by use of the multistate Bennet acceptance ratio MBAR method [43]. To achieve a more robust estimation of free energies, each simulation was repeated multiple times, using different initial velocities drawn from the Maxwell-Boltzmann distribution. Unless otherwise mentioned, the reported binding free energies are the mean of three runs, and statistical uncertainties are given one standard error of the mean.

As descripted in Ref. [13] for each model a population distribution for the determination coefficient $R^{2}$, the mean unsigned error MUE and the Kendall $\tau$ parameters was computed by bootstrapping each free energy predictions for each host-guest dataset for ten thousand times. The resulting distributions may not be symmetric around the mean, thus uncertainties are reported with a $95 \%$ confidence interval.

Additionally, for the SAMPLing leg of the challenge, binding free energies were evaluated using ModelB by skipping the first $1.5 \mathrm{~ns}$ of each window, and using $1-100 \%$ of the rest of the dataset. Uncertainties were taken as the standard deviation output from pymbar and were propagated to obtain an uncertainty for the reported standard free energy of binding. The total wall-clock time was also estimated by summing up the wall-clock time for each $\lambda$ window, in each phase and simulated process. The number of iterations was retrieved as the sum of the number of time-steps for each simulated process. For each host-guest replica 459,995,400 energy evaluations were carried on with an average wallclock time of $245 \mathrm{~h}$ for $C B 8$ systems and $190 \mathrm{~h}$ for $O A$. All input files for the SAMPL6 and SAMPLing protocols are publically available in the repository https://github.com/ michellab/SAMPL6inputs.

\section{Results}

\section{SAMPL6 challenge}

Results for the full SAMPL6 dataset are shown in Fig. 3 for each model without and with a buffer setup. As judged by mean unsigned error, ModelA/no-buffer is the least accurate protocol, with a MUE value ca. $5.7 \mathrm{kcal} \mathrm{mol}^{-1}$. ModelA/buffer offers small improvements, with the MUE decreasing to ca. $5.1 \mathrm{kcal} \mathrm{mol}^{-1}$. Addition of long-range dispersions and standard state correction terms in ModelB decreases errors further (MUE ca. 3.9 and $3.4 \mathrm{kcal} \mathrm{mol}^{-1}$ for the no-buffer and buffer setups respectively). ModelC improves over ModelB with MUE values ca. 1.4 and $1.6 \mathrm{kcal} \mathrm{mol}^{-1}$ for the no-buffer and buffer setups respectively. Thus, the additional counter-ions in the buffer setup improve accuracy for ModelA and ModelB but not ModelC. This could be because the SAMPL5 calculations were carried out with a no-buffer setup [13], and the empirical correction terms used in ModelC do not transfer to a buffer setup.

Ranking of the protocols according to correlation with experimental data yields a different outcome. ModelA/nobuffer and ModelB/no-buffer perform similarly well with $R^{2}$ and $\tau$ values ca. 0.6 , and a small decrease in predictive power is observed for ModelC/no-buffer but this is only significant for $R^{2}$. This drop is observed because the empirical correction term works well to bring the OA host-guest binding energies in line with the experimental values, but leads to a tendency to underestimate the CB8 binding energies. The use of a buffer also appears detrimental to predictive power, with all buffer protocols giving significant decreases in $R^{2}$ and $\tau$ parameters with respect to the equivalent no-buffer protocol.

Inspection of the results for the OA subset (Tables 1, 2) shows that ModelB and ModelC significantly improve the MUE over ModelA but not for $R^{2}$ or $\tau$ metrics that are ca. 0.7 and 0.5 respectively. The buffer protocol worsens MUE over the no-buffer protocol but does not influence predictive power. The same picture holds for the TEMOA subset, with improvements for MUE only observed upon switching from ModelA to ModelB and ModelC. Switching from no-buffer to buffer gives significant worsening of the MUE for ModelA and ModelB. The $R^{2}$ and $\tau$ metrics are high throughout (ca. 0.9 and 0.8) and insensitive to the various protocols. For the CB8 subset dramatic improvements in MUE are also observed as correction term are introduced (ModelA/no-buffer MUE ca. $7.3 \mathrm{kcal} \mathrm{mol}^{-1} \mathrm{vs.}$ ModelC/no-buffer MUE ca. $1.6 \mathrm{kcal} \mathrm{mol}^{-1}$ ). Unlike for the octa-acid guests switching from a no-buffer to buffer setup significantly improves the MUE for ModelA and ModelB, but not for ModelC where the MUE worsens. Thus, the buffer effects are host-guest dependent. For the OA and TEMOA hosts, the guests are negatively charged acids and explicit modelling of a buffer favors the binding process (average change in binding energies of $-0.9 \mathrm{kcal} \mathrm{mol}^{-1}$ for ModelB). For the CB8 host, the guests are positively charged amines and explicit modelling of a buffer disfavors the binding process (average change in binding energies of $+3.1 \mathrm{kcal} \mathrm{mol}^{-1}$ for ModelB). The effect is particularly pronounced for some $\mathrm{CB} 8$ guests, e.g. the binding energies of G3, G4 and G7 increase by more than $4 \mathrm{kcal} \mathrm{mol}^{-1}$ upon switching from a no-buffer to buffer protocol. None of the models tested yield significant predictive power with $R^{2}$ and $\tau$ metrics ca. 0.1 .

The largest outliers for $C B 8$ are guests G3, G4, G5 and G8. In particular the binding free energies of G3, G5 and G8 are lower than the experimental data by about $10 \mathrm{kcal} \mathrm{mol}^{-1}$ with ModelA/no-buffer or ModelB/no-buffer. The statistical 
Fig. 3 Comparison of the predicted and measured binding free energies for a ModelA/nobuffer, b ModelA/buffer, c ModelB/no-buffer, d ModelB/buffer, e ModelC/nobuffer, $\mathbf{f}$ ModelC/buffer for the 27 host-guest systems. The grey line denotes perfect correlation between predictions and measurements, while the yellow shaded region indicates $\mathrm{a} \pm 1 \mathrm{kcal} \mathrm{mol}^{-1}$ error bound. OA systems are colored in blue, TEMOA in green and CB8 in red
(A)

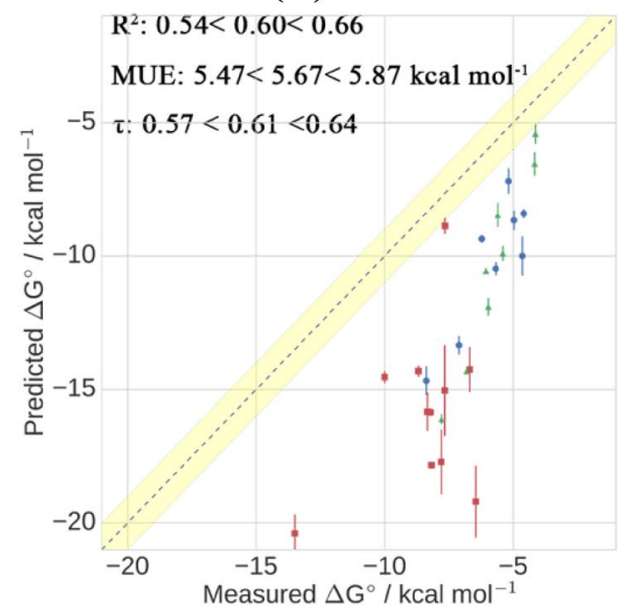

(C)

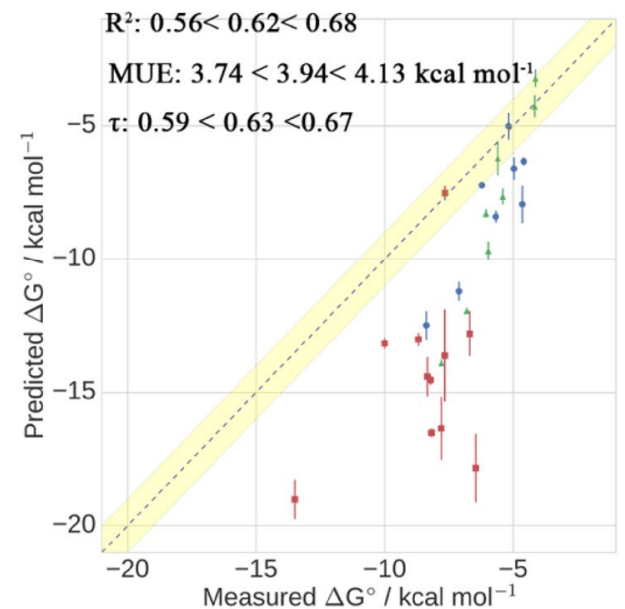

(E)

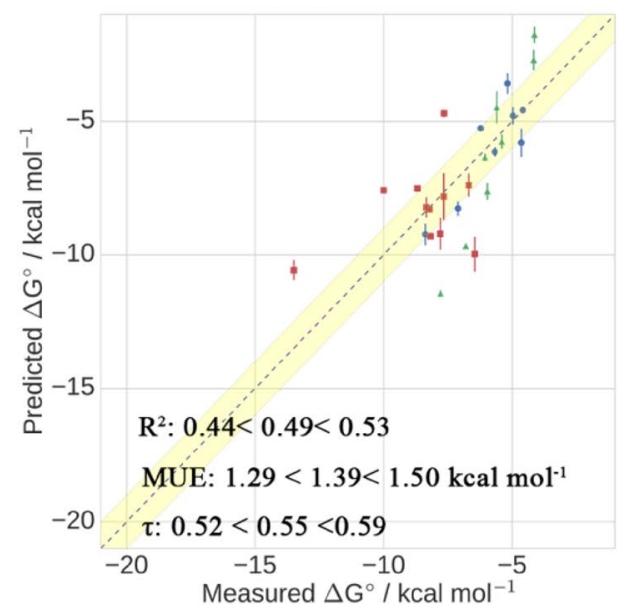

(B)

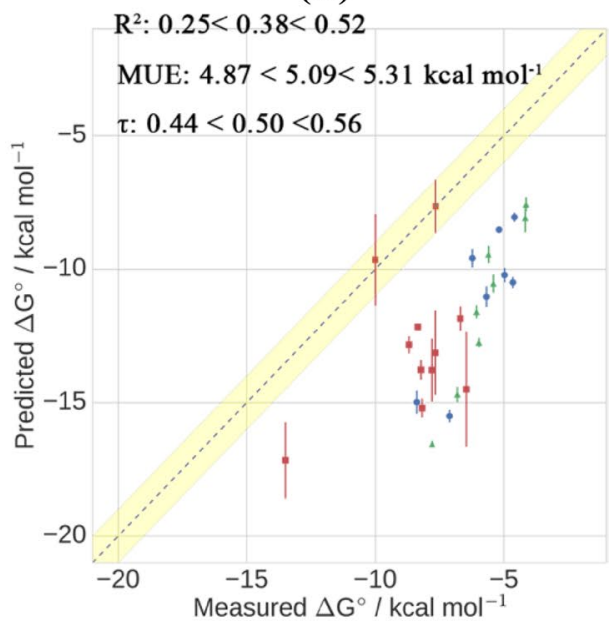

(D)

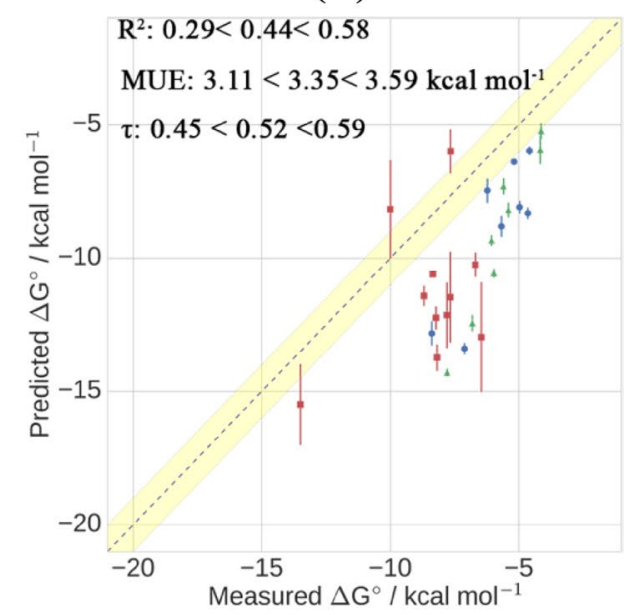

(F)

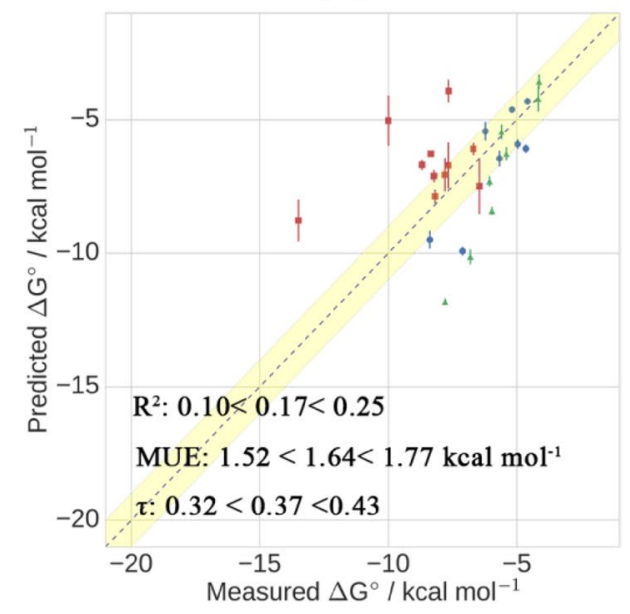

errors are also larger than for the octa-acids, suggesting greater challenges for converging free energy changes in $C B 8$ over the simulated time-scales. Switching to a buffer protocol decreases free energies of binding, and by up to ca. $5 \mathrm{kcal} \mathrm{mol}^{-1}$ for G3 and G8.
Among octa-acids the models correctly capture interesting trends in the experimental data. For instance, the models correctly predict that G7 binds significantly worse to TEMOA than to OA. The bulkiness of the two methyl groups $\beta$ to the carboxylic acid moiety hinders positioning 
Table 1 Results for all three models (no-buffer protocol) for individual host-guest families

\begin{tabular}{|c|c|c|c|c|}
\hline Guest & $\Delta G_{\text {bind }}$ ModelA & $\Delta G_{\text {bind }}$ ModelB & $\Delta G_{\text {bind }}$ ModelC & $\Delta G_{\text {bind }}$ experimental \\
\hline \multicolumn{5}{|l|}{$\mathrm{OA}$} \\
\hline G0 & $-10.5 \pm 0.3$ & $-8.4 \pm 0.2$ & $-6.1 \pm 0.2$ & $-5.7 \pm 0.1$ \\
\hline G1 & $-10.0 \pm 0.7$ & $-7.9 \pm 0.7$ & $-5.8 \pm 0.5$ & $-4.7 \pm 0.1$ \\
\hline $\mathrm{G} 2$ & $-14.7 \pm 0.5$ & $-12.5 \pm 0.5$ & $-9.2 \pm 0.4$ & $-8.4 \pm 0.1$ \\
\hline G3 & $-7.2 \pm 0.5$ & $-5.0 \pm 0.5$ & $-3.6 \pm 0.4$ & $-5.2 \pm 0.1$ \\
\hline G4 & $-13.3 \pm 0.3$ & $-11.2 \pm 0.4$ & $-8.3 \pm 0.3$ & $-7.1 \pm 0.1$ \\
\hline G5 & $-8.4 \pm 0.2$ & $-6.3 \pm 0.2$ & $-4.6 \pm 0.1$ & $-4.6 \pm 0.1$ \\
\hline G6 & $-8.7 \pm 0.4$ & $-6.6 \pm 0.4$ & $-4.8 \pm 0.3$ & $-5.0 \pm 0.1$ \\
\hline G7 & $-9.4 \pm 0.2$ & $-7.2 \pm 0.1$ & $-5.3 \pm 0.1$ & $-6.2 \pm 0.1$ \\
\hline $\mathrm{R}^{2}$ & $0.62<0.75<0.85$ & $0.62<0.73<0.84$ & $0.62<0.74<0.84$ & \\
\hline MUE & $4.17<4.41<4.66$ & $2.15<2.37<2.60$ & $0.65<0.82<1.00$ & \\
\hline$\tau$ & $0.43<0.54<0.64$ & $0.43<0.54<0.64$ & $0.43<0.54<0.64$ & \\
\hline \multicolumn{5}{|l|}{ TEMOA } \\
\hline G0 & $-10.6 \pm 0.1$ & $-8.3 \pm 0.2$ & $-6.4 \pm 0.2$ & $-6.1 \pm 0.1$ \\
\hline G1 & $-11.9 \pm 0.3$ & $-9.7 \pm 0.4$ & $-7.6 \pm 0.3$ & $-6.0 \pm 0.1$ \\
\hline $\mathrm{G} 2$ & $-14.3 \pm 0.1$ & $-11.9 \pm 0.1$ & $-9.7 \pm 0.1$ & $-6.8 \pm 0.1$ \\
\hline G3 & $-8.5 \pm 0.5$ & $-6.2 \pm 0.7$ & $-4.5 \pm 0.2$ & $-5.6 \pm 0.1$ \\
\hline G4 & $-16.1 \pm 0.2$ & $-13.9 \pm 0.1$ & $-11.4 \pm 0.1$ & $-7.8 \pm 0.1$ \\
\hline G5 & $-6.5 \pm 0.4$ & $-4.3 \pm 0.4$ & $-2.7 \pm 0.4$ & $-4.2 \pm 0.1$ \\
\hline G6 & $-9.9 \pm 0.3$ & $-7.6 \pm 0.3$ & $-5.8 \pm 0.3$ & $-5.4 \pm 0.1$ \\
\hline G7 & $-5.4 \pm 0.4$ & $-3.2 \pm 0.3$ & $-1.8 \pm 0.3$ & $-4.1 \pm 0.1$ \\
\hline $\mathrm{R}^{2}$ & $0.90<0.93<0.96$ & $0.89<0.93<0.97$ & $0.91<0.94<0.96$ & \\
\hline MUE & $4.47<4.66<4.84$ & $2.50<2.67<2.84$ & $1.58<1.72<1.86$ & \\
\hline$\tau$ & $0.85<0.86<0.87$ & $0.78<0.85<0.86$ & $0.85<0.86<0.87$ & \\
\hline \multicolumn{5}{|l|}{ CB8 } \\
\hline G0 & $-14.3 \pm 0.9$ & $-12.8 \pm 0.9$ & $-7.4 \pm 0.4$ & $-6.7 \pm 0.1$ \\
\hline G1 & $-8.9 \pm 0.3$ & $-7.5 \pm 0.3$ & $-4.7 \pm 0.1$ & $-7.7 \pm 0.1$ \\
\hline $\mathrm{G} 2$ & $-15.0 \pm 1.7$ & $-13.6 \pm 1.7$ & $-7.8 \pm 0.9$ & $-7.7 \pm 0.1$ \\
\hline G3 & $-19.2 \pm 1.4$ & $-17.8 \pm 1.3$ & $-10.0 \pm 0.7$ & $-6.5 \pm 0.1$ \\
\hline G4 & $-17.7 \pm 1.2$ & $-16.4 \pm 1.2$ & $-9.2 \pm 0.6$ & $-7.8 \pm 0.1$ \\
\hline G5 & $-17.8 \pm 0.1$ & $-16.5 \pm 0.2$ & $-9.3 \pm 0.1$ & $-8.2 \pm 0.1$ \\
\hline G6 & $-15.8 \pm 0.7$ & $-14.4 \pm 0.7$ & $-8.2 \pm 0.4$ & $-8.3 \pm 0.1$ \\
\hline G7 & $-14.5 \pm 0.2$ & $-13.2 \pm 0.2$ & $-7.6 \pm 0.1$ & $-10.0 \pm 0.1$ \\
\hline G8 & $-20.4 \pm 0.7$ & $-19.0 \pm 0.7$ & $-10.6 \pm 0.4$ & $-13.5 \pm 0.1$ \\
\hline G9 & $-14.3 \pm 0.2$ & $-13.0 \pm 0.3$ & $-7.5 \pm 0.1$ & $-8.7 \pm 0.1$ \\
\hline G10 & $-15.9 \pm 0.2$ & $-14.5 \pm 0.2$ & $-8.3 \pm 0.1$ & $-8.2 \pm 0.1$ \\
\hline $\mathrm{R}^{2}$ & $0.04<0.12<0.23$ & $0.04<0.12<0.23$ & $0.04<0.12<0.23$ & \\
\hline MUE & $6.90<7.33<7.76$ & $5.57<5.99<6.42$ & $1.40<1.57<1.76$ & \\
\hline$\tau$ & $-0.09<0.04<0.20$ & $-0.09<0.05<0.20$ & $-0.09<0.05<0.20$ & \\
\hline
\end{tabular}

of the guest in the smaller TEMOA cavity (Fig. 1a). The most significant outlier is G2 for which the models are unable to reproduce the significantly decreased binding energetics for TEMOA versus OA. A possible reason for this discrepancy is that the different ring puckering motions of the cyclohexenyl moiety in G2 may have been poorly sampled with the simulation protocols employed here.

\section{SAMPLing challenge}

Convergence plots for the calculated binding free energies of the three host-guests CB8-G3, OA-G3 and OA-G6 are presented in Fig. 4. ${ }^{1}$ Figure 4 a shows that for CB8-G3 the

\footnotetext{
$\overline{1}$ The SAMPLing free energies submitted on 01/19/2018 (5732q) were incorrectly evaluated due to a software bug. The results reported in this manuscript have been obtained after closure of the competition.
} 
Table 2 Results for all three models (buffer protocol) for individual host-guest families

\begin{tabular}{|c|c|c|c|c|}
\hline Guest & $\Delta G_{\text {bind }}$ ModelA & $\Delta G_{\text {bind }}$ ModelB & $\Delta G_{\text {bind }}$ ModelC & $\Delta G_{\text {bind }}$ experimental \\
\hline \multicolumn{5}{|l|}{$\mathrm{OA}$} \\
\hline G0 & $-11.0 \pm 0.4$ & $-8.8 \pm 0.4$ & $-6.5 \pm 0.3$ & $-5.7 \pm 0.1$ \\
\hline G1 & $-10.0 \pm 0.2$ & $-8.3 \pm 0.2$ & $-6.1 \pm 0.2$ & $-4.7 \pm 0.1$ \\
\hline $\mathrm{G} 2$ & $-15.0 \pm 0.4$ & $-12.8 \pm 0.5$ & $-9.5 \pm 0.3$ & $-8.4 \pm 0.1$ \\
\hline G3 & $-8.5 \pm 0.1$ & $-6.4 \pm 0.1$ & $-4.6 \pm 0.0$ & $-5.2 \pm 0.1$ \\
\hline G4 & $-15.5 \pm 0.2$ & $-13.4 \pm 0.2$ & $-9.9 \pm 0.2$ & $-7.1 \pm 0.1$ \\
\hline G5 & $-8.1 \pm 0.2$ & $-6.0 \pm 0.2$ & $-4.3 \pm 0.1$ & $-4.6 \pm 0.1$ \\
\hline G6 & $-10.2 \pm 0.3$ & $-8.1 \pm 0.2$ & $-5.9 \pm 0.2$ & $-5.0 \pm 0.1$ \\
\hline G7 & $-9.6 \pm 0.3$ & $-7.5 \pm 0.5$ & $-5.4 \pm 0.4$ & $-6.2 \pm 0.1$ \\
\hline $\mathrm{R}^{2}$ & $0.67<0.74<0.80$ & $0.63<0.71<0.78$ & $0.63<0.71<0.78$ & \\
\hline MUE & $4.97<5.14<5.31$ & $2.87<3.05<3.23$ & $0.95<1.08<1.22$ & \\
\hline$\tau$ & $0.50<0.56<0.64$ & $0.43<0.52<0.64$ & $0.43<0.52<0.64$ & \\
\hline \multicolumn{5}{|c|}{ TEMOA } \\
\hline G0 & $-11.6 \pm 0.2$ & $-9.3 \pm 0.2$ & $-7.3 \pm 0.2$ & $-6.1 \pm 0.1$ \\
\hline G1 & $-12.8 \pm 0.2$ & $-10.6 \pm 0.2$ & $-8.4 \pm 0.2$ & $-6.0 \pm 0.1$ \\
\hline $\mathrm{G} 2$ & $-14.7 \pm 0.3$ & $-12.4 \pm 0.3$ & $-10.1 \pm 0.3$ & $-6.8 \pm 0.1$ \\
\hline G3 & $-9.5 \pm 0.3$ & $-7.3 \pm 0.3$ & $-5.5 \pm 0.3$ & $-5.6 \pm 0.1$ \\
\hline G4 & $-16.6 \pm 0.1$ & $-14.3 \pm 0.1$ & $-11.8 \pm 0.1$ & $-7.8 \pm 0.1$ \\
\hline G5 & $-8.1 \pm 0.5$ & $-5.9 \pm 0.5$ & $-4.2 \pm 0.5$ & $-4.2 \pm 0.1$ \\
\hline G6 & $-10.5 \pm 0.3$ & $-8.2 \pm 0.3$ & $-6.3 \pm 0.3$ & $-5.4 \pm 0.1$ \\
\hline G7 & $-7.6 \pm 0.3$ & $-5.2 \pm 0.3$ & $-3.6 \pm 0.3$ & $-4.1 \pm 0.1$ \\
\hline $\mathrm{R}^{2}$ & $0.89<0.92<0.95$ & $0.89<0.93<0.96$ & $0.89<0.93<0.96$ & \\
\hline MUE & $5.48<5.66<5.84$ & $3.23<3.41<3.59$ & $1.51<1.63<1.77$ & \\
\hline$\tau$ & $0.78<0.84<0.85$ & $0.78<0.85<0.86$ & $0.78<0.84<0.85$ & \\
\hline \multicolumn{5}{|l|}{ CB8 } \\
\hline G0 & $-11.9 \pm 0.5$ & $-10.2 \pm 0.5$ & $-6.1 \pm 0.2$ & $-6.7 \pm 0.1$ \\
\hline G1 & $-7.6 \pm 1.0$ & $-6.0 \pm 0.8$ & $-3.9 \pm 0.4$ & $-7.7 \pm 0.1$ \\
\hline G2 & $-13.1 \pm 1.6$ & $-11.5 \pm 1.7$ & $-6.7 \pm 0.9$ & $-7.7 \pm 0.1$ \\
\hline G3 & $-14.5 \pm 2.2$ & $-13.0 \pm 2.1$ & $-7.5 \pm 1.1$ & $-6.5 \pm 0.1$ \\
\hline G4 & $-13.8 \pm 1.2$ & $-12.1 \pm 1.2$ & $-7.1 \pm 0.6$ & $-7.8 \pm 0.1$ \\
\hline G5 & $-15.2 \pm 0.4$ & $-13.7 \pm 0.5$ & $-7.9 \pm 0.3$ & $-8.2 \pm 0.1$ \\
\hline G6 & $-12.2 \pm 0.1$ & $-10.6 \pm 0.0$ & $-6.3 \pm 0.0$ & $-8.3 \pm 0.1$ \\
\hline G7 & $-9.7 \pm 1.7$ & $-8.2 \pm 1.8$ & $-5.0 \pm 0.9$ & $-10.0 \pm 0.1$ \\
\hline G8 & $-17.2 \pm 1.4$ & $-15.5 \pm 1.5$ & $-8.8 \pm 0.8$ & $-13.5 \pm 0.1$ \\
\hline G9 & $-12.3 \pm 0.3$ & $-11.4 \pm 0.4$ & $-6.7 \pm 0.2$ & $-8.7 \pm 0.1$ \\
\hline G10 & $-13.8 \pm 0.4$ & $-12.2 \pm 0.4$ & $-7.1 \pm 0.2$ & $-8.2 \pm 0.1$ \\
\hline $\mathrm{R}^{2}$ & $0.00<0.13<0.33$ & $0.00<0.13<0.35$ & $0.00<0.13<0.35$ & \\
\hline MUE & $4.07<4.59<5.13$ & $2.96<3.52<4.08$ & $1.78<2.06<2.34$ & \\
\hline$\tau$ & $-0.12<0.06<0.27$ & $-0.09<0.10<0.30$ & $-0.09<0.10<0.30$ & \\
\hline
\end{tabular}

binding free energy estimate obtained using the full simulation dataset is $-13.8 \pm 0.7 \mathrm{kcal} \mathrm{mol}^{-1}$. Although the uncertainties are high the mean free energy rapidly settles around $-14 \mathrm{kcal} \mathrm{mol}^{-1}$ and similar estimates would have been obtained with about $20 \%$ of the simulation duration. The calculated binding free energies are consistent with those obtained for this host-guest with the SAMPL6 protocol $\left(-13.0 \pm 2.1 \mathrm{kcal} \mathrm{mol}^{-1}\right.$, Table 2). The SAMPLing reference binding free energy computed by the organizers using the software YANK is significantly different and more precise $\left(-10.8 \pm 0.2 \mathrm{kcal} \mathrm{mol}^{-1}\right)$ [56]. The reference value is also in better agreement with experimental data, though substantial differences remain $\left(-6.5 \pm 0.1 \mathrm{kcal} \mathrm{mol}^{-1}\right)$. It appears at least $60 \%$ of the simulation duration is needed to eliminate drifts in the running average for the reference calculation.

For OA-G3 (Fig. 4b) the binding free energies computed with SOMD and by the organizers are similarly precise and converge to $-5.7 \pm 0.1 \mathrm{kcal} \mathrm{mol}^{-1}$ and $-6.7 \pm 0.1 \mathrm{kcal} \mathrm{mol}^{-1}$ respectively. The SOMD SAMPLing free energies are as precise but more 


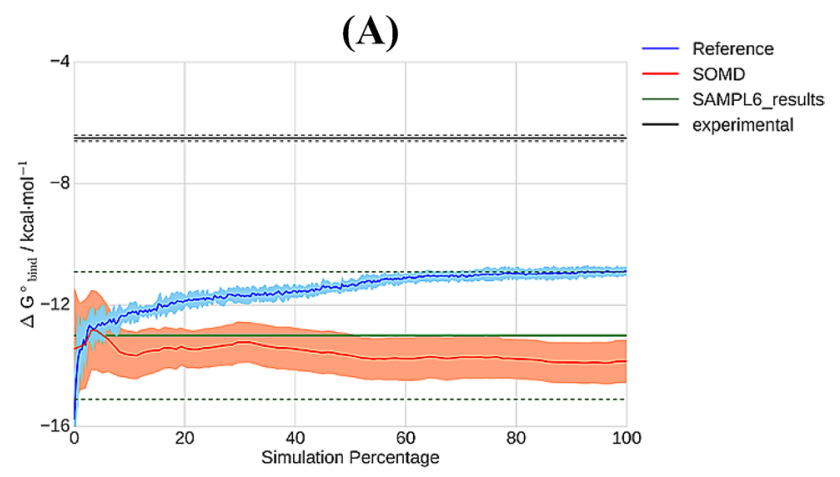

(B)

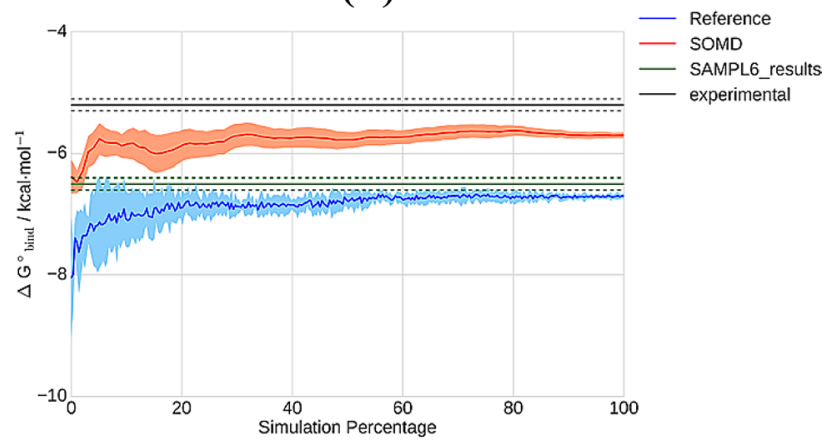

(C)

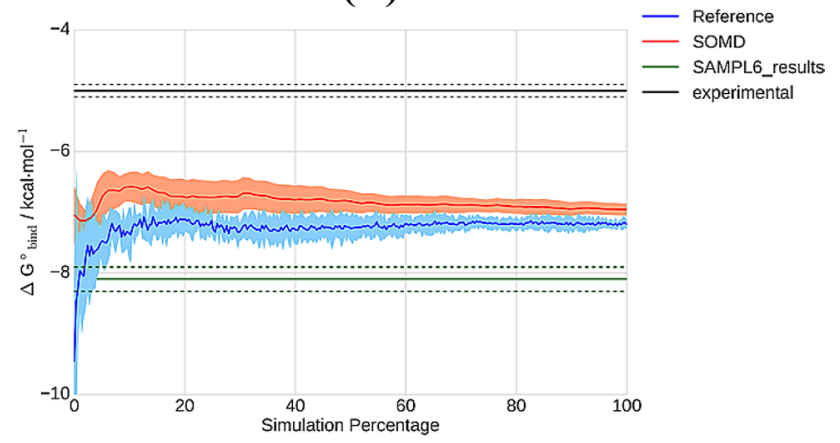

Fig. 4 Comparison of standard binding free energies computed with SOMD (red) to SAMPLing reference values (blue) for $C B 8-G 3$ (a), $O A-G 3$ (b) and $O A-G 6$ (c). Bold lines denote the average free energy from five replicate simulations started from different coordinates. Shaded areas denote $\pm 1 \sigma$. The SAMPL6 and experimental results are depicted with green and black lines respectively, and the dotted lines denote $\pm 1 \sigma$

accurate than the SOMD SAMPL6 free energies $\left(-6.4 \pm 0.1 \mathrm{kcal} \mathrm{mol}^{-1}\right.$, Table 2$)$ in comparison with experimental data $\left(-5.2 \pm 0.1 \mathrm{kcal} \mathrm{mol}^{-1}\right)$. The running average for both protocols is stable after ca. $20 \%$ of the simulation duration. For OA-G6 the SOMD and organizer's free energies rapidly converge to very similar values $\left(-6.9 \pm 0.1 \mathrm{kcal} \mathrm{mol}^{-1}\right.$ vs. $-7.1 \pm 0.1 \mathrm{kcal} \mathrm{mol}^{-1}$ respectively). These figures are in better agreement with experiment $\left(-5.0 \pm 0.1 \mathrm{kcal} \mathrm{mol}^{-1}\right)$ than the SAMPL6 SOMD free energies $\left(-8.1 \pm 0.2 \mathrm{kcal} \mathrm{mol}^{-1}\right)$.

Overall comparison of free energies estimated from the SAMPL6 and SAMPLing protocols shows that averaging results over multiple starting host-guest structures improved agreement of predictions with experiment for OA-G3 and OA-G6 but not CB8. No clear reason emerges to explain differences in binding free energies computed by SOMD and YANK.

\section{Conclusions}

AFE calculations were employed to estimate standard binding free energies for 27 host-guests in the SAMPL6 competition. Protocols similar to that used in the SAMPL5 competition were adopted (ModelA/no-buffer and ModelB/no-buffer) [13], leading to results of comparable performance to SAMPL5 (SAMPL6 ModelB/no-buffer $R^{2}$ ca. 0.6, MUE $3.9 \mathrm{kcal} \mathrm{mol}^{-1}, N=27$ vs. SAMPL5 ModelC $R^{2}$ ca. 0.7, MUE $3.4 \mathrm{kcal} \mathrm{mol}^{-1}, N=22$ ). The reasons for the systematic overestimation of free energies of binding remain unclear; this could be because of a neglect of long-range correction term to electrostatics, or use of non-polarizable force-fields.

Additionally, an empirical correction term derived by a linear regression approach against SAMPL5 data was devised to correct for systematic errors in the free energy calculation protocol (Model C/no-buffer). This leads to significant improvements in mean-unsigned error but a slight decrease in correlation with experimental trends (MUE ca. $1.4 \mathrm{kcal} \mathrm{mol}^{-1}, \mathrm{R}^{2}$ ca. 0.5 ). High accuracy predictions and correlations with experimental data were achieved for the OA and TEMOA hosts, but CB8 proved more challenging, with significantly higher uncertainties in the computed binding free energies and poor correlation with experiment.

The influence of the modelled buffer on the computed binding free energies was also investigated. The main finding is that explicit modelling of the buffer enhances binding of negatively charged guests to OA and TEMOA, and weakens binding of positively charged guests to CB8. Overall the MUE for the dataset (ModelA and ModelB) decreases by about $0.6 \mathrm{kcal} \mathrm{mol}^{-1}$ because the CB8 binding energies are more in line with experimental data. However, this improvement is also accompanied by a drop of ca. 0.2 in $\mathrm{R}^{2}$. The empirical correction term derived against SAMPL5 data is incompatible with a protocol that models explicitly a buffer, presumably because no buffer was modelled in the SAMPL5 calculations [13].

With respect to other SAMPL6 submissions the results obtained with SOMD were encouraging and among the top performing models for OA and TEMOA as judged 
by $\mathrm{R}^{2}$ and MUE metrics. CB8 proved challenging for most participating groups. SOMD ModelC/no-buffer gave the lowest MUE values among all submissions (ca. $1.5 \mathrm{kcal} \mathrm{mol}^{-1}$ ), but the predictive power was insignificant $\left(\mathrm{R}^{2}\right.$ ca. 0.1) [31].

The OA-G3 and OA-G6 binding free energies computed with the SAMPLing protocol were significantly different from those computed with SAMPL6 protocol (0.7 and $1.2 \mathrm{kcal} \mathrm{mol}^{-1}$ respectively). A standard practice in our group is to at least estimate uncertainties in computed binding free energies from triplicate runs initiated from the same input coordinates. This gives a reasonable estimate of the extent to which free energies are reproducible given a starting condition, but can also give a misleading impression of convergence. Where multiple reasonable poses can be produced, efforts are better spent evaluating free energies with simulations started from different input coordinates. Comparison of SOMD's free energies with the reference values (YANK) provided by the organizers yields a mixed picture, with a substantially significant difference (CB8-G3, $3 \pm 0.7 \mathrm{kcal} \mathrm{mol}^{-1}$ ), a moderate difference $\left(\mathrm{OA}-\mathrm{G} 3,1 \pm 0.2 \mathrm{kcal} \mathrm{mol}^{-1}\right)$, and an insignificant difference (OA-G6 $0.2 \pm 0.2 \mathrm{kcal} \mathrm{mol}^{-1}$ ). There are several algorithmic differences between the two codes that could explain discrepancies, a notable one being an atom-based Barker-Watts reaction-field treatment of long-range electrostatics (SOMD) versus PME (YANK). Other differences exist around the treatment of soft-cores, the coupling of non-bonded and bonded interactions with the $\lambda$ schedule, and electrostatic correction terms for charged guests. More systematic reproducibility studies on larger datasets will be needed to isolate the factors that contribute to the observed variability. Such efforts are important to validate the robustness and transferability of molecular simulation algorithms.

Acknowledgements Julien Michel is supported by a University Research Fellowship from the Royal Society. The research leading to these results has received funding from the European Research Council under the European Union's Seventh Framework Programme (FP7/2007-2013)/ERC Grant Agreement No. 336289.

Open Access This article is distributed under the terms of the Creative Commons Attribution 4.0 International License (http://creativeco mmons.org/licenses/by/4.0/), which permits unrestricted use, distribution, and reproduction in any medium, provided you give appropriate credit to the original author(s) and the source, provide a link to the Creative Commons license, and indicate if changes were made.

\section{References}

1. Jorgensen WL (2004) The many roles of computation in drug discovery. Science 303(5665):1813-1818
2. Michel J (2014) Current and emerging opportunities for molecular simulations in structure-based drug design. Phys Chem Chem Phys 16(10):4465-4477

3. Larson SM, Snow CD, Shirts M, Pande VS (2009) Folding@ Home and Genome@Home: using distributed computing to tackle previously intractable problems in computational biology. ArXiv09010866 Phys. Q-Bio

4. Shaw DE, Dror RO, Salmon JK, Grossman JP, Mackenzie KM, Bank JA, Young C, Deneroff MM, Batson B, Bowers KJ et al (2009) Millisecond-scale molecular dynamics simulations on anton. In Proceedings of the conference on high performance computing networking, storage and analysis; SC'09; ACM, New York, pp 39:1-39:11

5. Kohlhoff K, Shukla D, Lawrenz M, Bowman G, Konerding D, Belov D, Altman R, Pande V (2014) Cloud-Based simulations on Google Exacycle Reveal ligand modulation of GPCR activation pathways. Nat Chem 6:15

6. Michel J, Foloppe N, Essex JW (2010) Rigorous free energy calculations in structure-based drug design. Mol Inform 29(8-9):570-578

7. Deng Y, Roux B (2006) Calculation of standard binding free energies: aromatic molecules in the T4 lysozyme L99A mutant. J Chem Theory Comput 2(5):1255-1273

8. Chang C-E, Gilson MK (2004) Free energy, entropy, and induced fit in host-guest recognition: calculations with the second-generation mining minima algorithm. J Am Chem Soc 126(40):13156-13164

9. Mey ASJS, Juárez-Jiménez J, Michel J (2017) Impact of domain knowledge on blinded predictions of binding energies by alchemical free energy calculations. J Comput Aided Mol Des 32:199-210. https://doi.org/10.1007/s10822-017-0083-9

10. Calabrò G, Woods CJ, Powlesland F, Mey ASJS, Mulholland AJ, Michel J (2016) Elucidation of nonadditive effects in protein-ligand binding energies: thrombin as a case study. J Phys Chem B 120(24):5340-5350

11. Wang L, Wu Y, Deng Y, Kim B, Pierce L, Krilov G, Lupyan D, Robinson S, Dahlgren MK, Greenwood J et al (2015) Accurate and reliable prediction of relative ligand binding potency in prospective drug discovery by way of a modern freeenergy calculation protocol and force field. J Am Chem Soc 137(7):2695-2703

12. Aldeghi M, Heifetz A, Bodkin MJ, Knapp S, Biggin PC (2015) Accurate calculation of the absolute free energy of binding for drug molecules. Chem Sci 7(1):207-218

13. Bosisio S, Mey ASJS, Michel J (2017) Blinded predictions of host-guest standard free energies of binding in the SAMPL5 challenge. J Comput Aided Mol Des 31(1):61-70

14. Bosisio S, Mey ASJS, Michel J (2016) Blinded predictions of distribution coefficients in the SAMPL5 challenge. J Comput Aided Mol Des 30(11):1101-1114

15. Bannan CC, Burley KH, Chiu M, Shirts MR, Gilson MK, Mobley DL (2016) Blind prediction of cyclohexane-water distribution coefficients from the SAMPL5 challenge. J Comput Aided Mol Des 30(11):927-944

16. Rodil A, Bosisio S, Ayoup MS, Quinn L, Cordes DB, Slawin AMZ, Murphy CD, Michel J, O'Hagan D (2018) Metabolism and hydrophilicity of the polarised 'Janus Face' all- $c$ is tetrafluorocyclohexyl ring, a candidate motif for drug discovery. Chem Sci 9(11):3023-3028

17. Chodera JD, Mobley DL, Shirts MR, Dixon RW, Branson K, Pande VS (2011) Alchemical free energy methods for drug discovery: progress and challenges. Curr Opin Struct Biol 21(2): $150-160$

18. Chen I-J, Foloppe N (2011) Is conformational sampling of druglike molecules a solved problem? Drug Dev Res 72(1):85-94 
19. Souaille M, Roux B (2001) Extension to the weighted histogram analysis method: combining umbrella sampling with free energy calculations. Comput Phys Commun 135:40-57

20. Li H, Fajer M, Yang W (2007) Simulated scaling method for localized enhanced sampling and simultaneous "alchemical" free energy simulations: a general method for molecular mechanical, quantum mechanical, and quantum mechanical $/$ molecular mechanical simulations. J Chem Phys 126(2):024106

21. Halgren TA, Damm W (2001) Polarizable force fields. Curr Opin Struct Biol 11(2):236-242

22. Kastenholz MA, Hünenberger PH (2004) Influence of artificial periodicity and ionic strength in molecular dynamics simulations of charged biomolecules employing lattice-sum methods. J Phys Chem B 108(2):774-788

23. Reif Maria M, Oostenbrink C (2013) Net Charge changes in the calculation of relative ligand-binding free energies via classical atomistic molecular dynamics simulation. J Comput Chem 35(3):227-243

24. Rocklin GJ, Mobley DL, Dill KA, Hünenberger PH (2013) Calculating the binding free energies of charged species based on explicit-solvent simulations employing lattice-sum methods: an accurate correction scheme for electrostatic finite-size effects. J Chem Phys 139(18):184103

25. Mey ASJS, Juárez-Jiménez J, Hennessy A, Michel J (2016) Blinded predictions of binding modes and energies of HSP90- $\alpha$ ligands for the 2015 D3R Grand Challenge. Bioorg Med Chem 24(20):4890-4899

26. Gaieb Z, Liu S, Gathiaka S, Chiu M, Yang H, Shao C, Feher VA, Walters WP, Kuhn B, Rudolph MG et al (2018) D3R Grand Challenge 2: blind prediction of protein-ligand poses, affinity rankings, and relative binding free energies. J Comput Aided Mol Des 32(1): $1-20$

27. Nicholls A, Mobley DL, Guthrie JP, Chodera JD, Bayly CI, Cooper MD, Pande VS (2008) Predicting small-molecule solvation free energies: an informal blind test for computational chemistry. J Med Chem 51(4):769-779

28. Mobley DL, Liu S, Cerutti DS, Swope WC, Rice JE (2012) Alchemical prediction of hydration free energies for SAMPL. J Comput Aided Mol Des 26(5):551-562

29. Peat TS, Dolezal O, Newman J, Mobley D, Deadman JJ (2014) Interrogating HIV integrase for compounds that Bind-a SAMPL challenge. J Comput Aided Mol Des 28(4):347-362

30. Mobley DL, Gilson MK (2017) Predicting binding free energies: frontiers and benchmarks. Annu Rev Biophys 46:531-558

31. Rizzi A, Murkli S, McNeill JN, Yao W, Sullivan M, Gilson MK, Chiu MW, Isaacs L, Gibb BC, Mobley DL et al (2018) Overview of the SAMPL6 host-guest binding affinity prediction challenge. bioRxiv. https://doi.org/10.1101/371724

32. Gan H, Benjamin CJ, Gibb BC (2011) Nonmonotonic assembly of a deep-cavity cavitand. J Am Chem Soc 133(13):4770-4773

33. Gibb CLD, Gibb BC (2014) Binding of cyclic carboxylates to octa-acid deep-cavity cavitand. J Comput Aided Mol Des 28(4):319-325

34. Sullivan MR, Sokkalingam P, Nguyen T, Donahue JP, Gibb BC (2017) Binding of carboxylate and trimethylammonium salts to octa-acid and TEMOA Deep-Cavity cavitands. J Comput Aided Mol Des 31(1):21-28

35. Gan H, Gibb BC (2013) Guest-mediated switching of the assembly state of a water-soluble deep-cavity cavitand. Chem Commun 49(14):1395-1397
36. Assaf KI, Nau WM (2014) Cucurbiturils: from synthesis to highaffinity binding and catalysis. Chem Soc Rev 44(2):394-418

37. Biedermann F, Scherman OA (2012) Cucurbit[8]uril mediated donor-acceptor ternary complexes: a model system for studying charge-transfer interactions. J Phys Chem B 116(9):2842-2849

38. Vázquez J, Remón P, Dsouza RN, Lazar AI, Arteaga JF, Nau WM, Pischel U (2014) A simple assay for quality binders to cucurbiturils. Chem - Eur J 20(32):9897-9901

39. Liu S, Ruspic C, Mukhopadhyay P, Chakrabarti S, Zavalij PY, Isaacs L (2005) The Cucurbit[n]uril family: prime components for self-sorting systems. J Am Chem Soc 127(45):15959-15967

40. Michel J, Essex JW (2010) Prediction of protein-ligand binding affinity by free energy simulations: assumptions, pitfalls and expectations. J Comput Aided Mol Des 24(8):639-658

41. Jorgensen WL, Buckner JK, Boudon S, Tirado-Rives J (1988) Efficient computation of absolute free energies of binding by computer simulations. Application to the methane dimer in water. J Chem Phys 89(6):3742-3746

42. Gilson MK, Given JA, Bush BL, McCammon JA (1997) The statistical-thermodynamic basis for computation of binding affinities: a critical review. Biophys J 72(3):1047-1069

43. Shirts MR, Mobley DL, Chodera JD, Pande VS (2007) Accurate and efficient corrections for missing dispersion interactions in molecular simulations. J Phys Chem B 111(45):13052-13063

44. McGann MFRED, Docking HYBRID (2012) Performance on standardized datasets. J Comput Aided Mol Des 26(8):897-906

45. McGann MFRED (2011) Pose prediction and virtual screening accuracy. J Chem Inf Model 51(3):578-596

46. Kelley BP, Brown SP, Warren GL, Muchmore SW (2015) POSIT: flexible shape-guided docking for pose prediction. J Chem Inf Model 55(8):1771-1780

47. ParmEd - ParmEd documentation. http://parmed.github.io/ ParmEd/html/index.html. Accessed 29 Mar 2018

48. Case D, Cerutti DS, Cheatham T, Darden T, Duke R, Giese TJ, Gohlke H, Götz A, Greene D, Homeyer N et al (2017) Amber 2017. University of California, San Francisco

49. Wang J, Wang W, Kollman PA, Case DA (2006) Automatic atom type and bond type perception in molecular mechanical calculations. J Mol Graph Model 25(2):247-260

50. Wang J, Wolf RM, Caldwell JW, Kollman PA, Case DA (2004) Development and testing of a general amber force field. J Comput Chem 25(9):1157-1174

51. Jorgensen WL, Chandrasekhar J, Madura JD, Impey RW, Klein ML (1983) Comparison of simple potential functions for simulating liquid water. J Chem Phys 79(2):926-935

52. Woods C, Mey A, Calabro G, Michel J (2016) Sire molecular simulations framework. https://www.siremol.org/

53. Eastman P, Friedrichs MS, Chodera JD, Radmer RJ, Bruns CM, Ku JP, Beauchamp KA, Lane TJ, Wang L-P, Shukla D et al (2013) OpenMM 4: a reusable, extensible, hardware independent library for high performance molecular simulation. J Chem Theory Comput 9(1):461-469

54. Andersen HC (1980) Molecular dynamics simulations at constant pressure and/or temperature. J Chem Phys 72(4):2384-2393

55. Tironi IG, Sperb R, Smith PE, van Gunsteren WF (1995) A generalized reaction field method for molecular dynamics simulations. J Chem Phys 102(13):5451-5459

56. https://github.com/choderalab/yank. Accessed 28 Aug 2018 